\title{
Identifying molecular markers suitable for Frl selection in tomato breeding
}

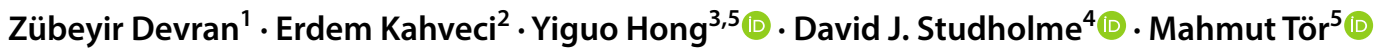

Received: 24 April 2018 / Accepted: 21 June 2018 / Published online: 7 July 2018

(C) The Author(s) 2018

\begin{abstract}
Modern plant breeding heavily relies on the use of molecular markers. In recent years, next generation sequencing (NGS) emerged as a powerful technology to discover DNA sequence polymorphisms and generate molecular markers very rapidly and cost effectively, accelerating the plant breeding programmes. A single dominant locus, $F r l$, in tomato provides resistance to the fungal pathogen Fusarium oxysporum f. sp. radicis-lycopersici (FORL), causative agent of Fusarium crown and root rot. In this study, we describe the generation of molecular markers associated with the $\mathrm{Frl}$ locus. An $\mathrm{F}_{2}$ mapping population between an FORL resistant and a susceptible cultivar was generated. NGS technology was then used to sequence the genomes of a susceptible and a resistant parent as well the genomes of bulked resistant and susceptible $\mathrm{F}_{2}$ lines. We zoomed into the $\mathrm{Frl}$ locus and mapped the locus to a $900 \mathrm{~kb}$ interval on chromosome 9. Polymorphic single-nucleotide polymorphisms (SNPs) within the interval were identified and markers co-segregating with the resistant phenotype were generated. Some of these markers were tested successfully with commercial tomato varieties indicating that they can be used for marker-assisted selection in large-scale breeding programmes.
\end{abstract}

\section{Introduction}

Cultivated tomato (Solanum lycopersicum L.) is the second most important consumed vegetables, grown worldwide and cultivated for fresh market and processed consumption (Foolad and Panthee 2012). As with many other crop plants,

Communicated by Hong-Qing Ling.

Electronic supplementary material The online version of this article (https://doi.org/10.1007/s00122-018-3136-0) contains supplementary material, which is available to authorized users.

Mahmut Tör

m.tor@worc.ac.uk

1 Faculty of Agriculture, Department of Plant Protection, University of Akdeniz, Antalya, Turkey

2 Department of Plant Pathology, M.Y. Genetik Agriculture Technology Laboratory, Antalya, Turkey

3 College of Life and Environmental Sciences, Research Centre for Plant RNA Signaling, Hangzhou Normal University, Hangzhou, China

4 Biosciences, College of Life and Environmental Sciences, University of Exeter, Exeter, UK

5 Institute of Science and the Environment, University of Worcester, Worcester WR2 6AJ, UK many pests and pathogens including viruses, nematodes, bacteria and fungi attack cultivated tomato varieties. Tomato vascular wilts and crown and root rot diseases are the most important devastating diseases and are caused by the fungal pathogens Fusarium oxysporum f. sp. lycopersici (FOL) and Fusarium oxysporum f. sp. radicis-lycopersici (FORL), respectively (Lievens et al. 2009). FORL has been reported to have a broader host range than FOL (Edel-Hermann et al. 2011) and is considered to cause significant yield losses in the greenhouse, open field crops and soilless production system. Although FORL is a soil-borne pathogen, dissemination of air-borne microconidia helps pathogen to re-infect the plants and cause an epidemic (Szczechura et al. 2013).

Several approaches to control FORL have been taken. These include: (a) pesticide applications such as using carbendazim (MBC) and the soil fumigant dazomet (DAZ) (Zhao et al. 2016); (b) use of biopesticides or biological control agents such as Bacillus species (Baysal et al. 2013); (c) soil solarization where pathogen infested soil is covered with polythene cover and the soil is subjected to high solar temperature (Saremi et al. 2008); and (d) use of resistant varieties such as those that contain the Frl gene (Fazio et al. 1999).

A comparison of these various control methods shows that the use of resistant varieties is highly desirable, cost-effective 
and environmentally safe. A genetic locus designated $\mathrm{Frl}$ provides resistance to FORL in tomato. Originally this locus was introduced into the cultivated S. lycopersicum from the wild species S. peruvianum (Fazio et al. 1999) and since then, it has been used in commercial plant breeding programmes.

Transcriptomics, proteomics and genetics studies have been performed to understand FORL-tomato interactions especially with tomato lines carrying $\mathrm{Frl}$ locus. Transcriptomic studies carried out with resistant and susceptible near isogenic lines revealed that in incompatible interactions, defence genes related to secondary metabolites and tryptophan metabolism showed elevated expression level, while in compatible interactions, increased level of gene expression related to oxidative burst and necrosis have been observed (Manzo et al. 2016).

Mazzeo et al. (2014) took a differential proteomic approach to investigate changes in resistant and susceptible tomato cultivars infected with FORL. Their results showed that there is accumulation of defence-related proteins including glutathione S-transferase in the resistant cultivar while proteins involved in redox reactions accumulated in susceptible cultivar.

Genetic studies showed that $\mathrm{Frl}$ is a single dominant locus on chromosome 9 and several molecular markers including the RFLP-based marker TG101 have been shown to be linked to Frl (Truong et al. 2011). As thousands of plant lines are screened in a given breeding programmes, markerassisted selection (MAS) offers advantages over classic phenotype-based selection. This is due to the fact that it can save time, resources and efforts and selection can be done at the seedling stages (Collard and Mackill 2008). Further molecular studies resulted in the development of RAPD (Truong et al. 2011) and SCAR (Mutlu et al. 2015) markers that are linked to the Frl. We have tried some of these markers but none of these have proven to be close enough to be successfully used in high throughput breeding programmes as they are several cM away from the $F r l$ locus allowing the high number of recombination events to occur, which is not desirable for commercial breeding activities.

Here, we used next-generation sequencing technology and bulk segregant analysis method to develop markers that are tightly linked to $\mathrm{Frl}$, MAS-friendly, easy to use and reliable. We report the generation of these markers and demonstrate that they can clearly separate the resistant and susceptible lines even in the available commercial lines.

\section{Materials and methods}

\section{Plant lines and mapping populations}

An $\mathrm{F}_{2}$ mapping population was generated from a cross between the susceptible (MT-7000) and resistant (MT-7028) tomato pure lines by breeders (Multi Tohum A.Ş., Antalya, Turkey) and was used in the experiments.

\section{Fungal isolate and pathology methods}

Fusarium oxysporum f.sp. radicis-lycopersici isolate, No: F-125 (M.Y. Genetik Tarım Teknoloji Laboratuvar Tic. Ltd. Şti, Antalya, Turkey), was maintained in $50 \%$ glycerol at $-80^{\circ} \mathrm{C}$ and was used throughout this study. The fungus was grown in Czapek Dox Broth on a rotary shaker for 7 days at $23{ }^{\circ} \mathrm{C}$. The broth culture was filtered through two layers of cheesecloth. The suspension was centrifuged at $3750 \mathrm{rpm}$ for $15 \mathrm{~min}$ and conidia were re-suspended in sterile $\mathrm{dH}_{2} 0$. The roots of seedlings at one true leaf stage were washed off substrate, dipped in a suspension of $10^{7}$ spores $/ \mathrm{ml}$. Seedlings were then transplanted in a sterilized mixture and kept in a growth chamber at $23{ }^{\circ} \mathrm{C}$ with a $12 \mathrm{~h}$ photoperiods for 30 days. Control plants were treated with sterile $\mathrm{dH}_{2} \mathrm{O}$ in a similar manner. At the end of incubation period, plants were evaluated as resistant or susceptible on the basis of existence of brown lesions on roots and crowns (Fazio et al. 1999; Xu et al. 2006).

\section{DNA isolation and genome sequencing}

Young leaves were collected from plants after pathotyping with FORL. Plant genomic DNA was then isolated using the Wizard Magnetic Kit (Promega) following the manufacturer's instructions. DNA was extracted separately from each individual of the $\mathrm{F}_{2}$ plant lines. The resistant and susceptible bulks were generated from twenty resistant and twenty susceptible $\mathrm{F}_{2}$ individuals, respectively, as described (Devran et al. 2015) and used for genomic sequencing. We generated 1 lane of 100 bp paired-end Illumina HiSeq 2500 sequencing data for each parent (resistant and susceptible) line and bulked (resistant and susceptible) pools.

\section{Bioinformatics and NGS analysis}

The Illumina reads were first trimmed based on their quality scores using BBDuk (filterk $=27$, trimk $=27$; http://jgi.doe. gov/data-and-tools/bb-tools/) to remove Illumina adapters and to quality-trim both ends to Q12. Frl was mapped previously on tomato chromosome 9 (https://solgenomics.net/ locus/566/view). We then used trimmed sequences from resistant and susceptible parents and resistant and susceptible bulks on the reference genome using Burrows-Wheeler Alignment tool (BWA) (Li and Durbin 2009). The region on chromosome 9:4205900-5108100 was extracted using SAMtools (Li et al. 2009) (https://sourceforge.net/proje cts/samtools/files/), and single-nucleotide variants (SNVs) between resistant and susceptible lines were identified using BCFtools (http://www.htslib.org/doc/bcftools.html) as 
described by Yemataw et al. (2018). The alignment results for the interval were visualized using Integrative Genomics Viewer (IGV) (Robinson et al. 2011) after converting to the BAM format (Li et al. 2009).

\section{Developing molecular markers}

Before SNPs were converted into PCR-based Cleaved amplified polymorphic sequences (CAPS) markers, polymorphic sites were confirmed both on parents and bulks. We then randomly selected candidates to cover the $1.2 \mathrm{Mb}$ region and the SNPs were converted into CAPS marker using dCAPS (http://helix.wustl.edu/dcaps/dcaps.html) (Neff et al. 2002). All PCR amplifications and digestion of PCR products with relevant restriction enzymes were performed by following manufacturers' instructions and were visualized as described (Devran et al. 2015).

\section{Confirmation of linkage between established and newly generated markers}

Newly generated PCR-based markers were first tested on parents to confirm the identified polymorphisms and then on a segregating $542 \mathrm{~F}_{2}$ lines. Marker genotyping data and the fungal disease phenotyping data were used to identify the $\mathrm{Frl}$ interval. Recombinant lines and the physical map covering the $\mathrm{Frl}$ region were used to narrow the interval for generation of new markers that could be used in the MASprogramme. Sequences of PCR-based markers will be provided upon request.

\section{Accession numbers}

Tomato reference genome sequence (Tomato Genome Consortium 2012) GenBank: GCA_000188115.2 Solyc2.50. Accession number for the tomato chromosome 9 used is CM001072. The accession number for Sequenced Read Archive (SRA) is SRP138888.

\section{Results}

\section{Frl segregates as a single locus}

The susceptible $S$. lycopersicum pure line MT-7000 was crossed with the resistant, MT-7028, line. The $\mathrm{F}_{1}$ generation showed resistance to FORL, indicating that resistance was dominant. A population of 542 segregating $\mathrm{F}_{2}$ individuals derived from the $F_{1}$ was then screened with the fungus. Disease symptoms, rotting in the tap roots, chocolate brown cankers appearing at the soil line in the susceptible plants, were clearly visible after 30 days (Fig. 1). The segregation ratio observed in this bioassay was 415:127 (resistant:susceptible, $3: 1$; with Chi square $=0.05$ and $P=0.05$ ). This suggested that a single resistance locus, $\mathrm{Frl}$, was controlling the resistance in this cross, confirming the previous findings and allowing the subsequent analysis.

\section{Fine mapping defines a $900 \mathrm{~kb}$ interval for the $\mathrm{Frl}$ locus}

DNA from twenty resistant and twenty susceptible $F_{2}$ lines was pooled in equal concentrations to make up the
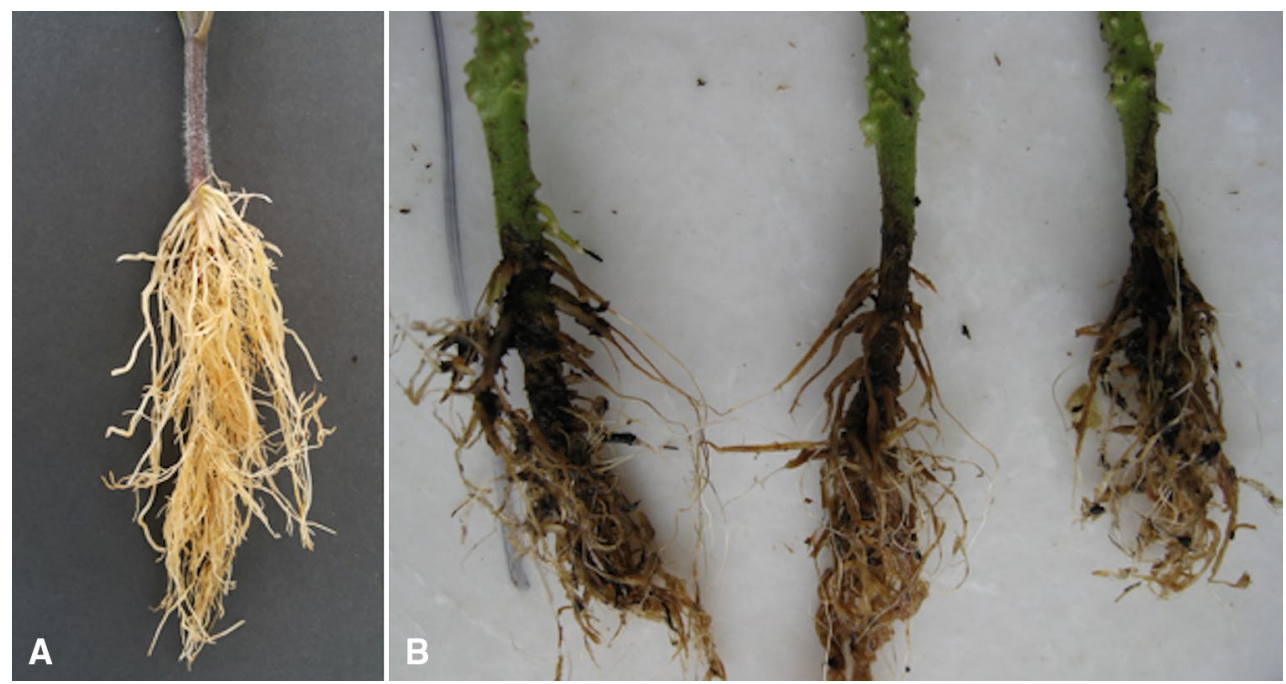

Fig. 1 Healthy and FORL-infected tomato roots. The roots of seedlings at one true leaf stage were washed off substrate, dipped in a suspension of $10^{7}$ spores $/ \mathrm{ml}$. Seedlings were then transplanted in a sterilized mixture and kept in a growth chamber at $23{ }^{\circ} \mathrm{C}$ with a $12 \mathrm{~h}$ photoperiods for 30 days. Control plants were treated with sterile $\mathrm{dH}_{2} \mathrm{O}$ in a similar manner. a Control roots, b FORL-infected roots 
resistant and susceptible bulks, respectively. We generated 100 bp paired-end Illumina HiSeq 2500 sequencing data from the two bulks (resistant and susceptible), comprising 46 million reads for the resistant and 42 million reads for the susceptible parent. Similarly, 80 million reads for the resistant parent and 118 million for the susceptible parent were generated. We then used the published tomato reference genome sequence (Tomato Genome Consortium 2012) (GenBank: GCA_000188115.2 Solyc2.50) as a reference to map sequence reads from both resistant and susceptible parents, and as well as sequences from both bulks. We concentrated on chromosome 9 around the previously published markers that were claimed to be linked to Frl (Truong et al. 2011; Mutlu et al. 2015). Initially, $1.2 \mathrm{Mb}$ region covering some of the previous markers was taken into account and some of the identified SNVs at the flanking regions were converted to CAPS marker and were then used to map the $\mathrm{Frl}$ locus. Once linkage was confirmed (Table 1), a total of $542 \mathrm{~F}_{2}$ lines were then screened with further markers and the $\mathrm{Frl}$ locus was fine mapped to a $900 \mathrm{~kb}$ interval on the reference genome between the molecular markers 4206 and $5108 \mathrm{~K}$ (Fig. 2). A gel image of segregating $\mathrm{F}_{2}$ lines was given as a representative of mapping Frl (Supplementary Figure 1).

\section{Interval contains single-nucleotide variants}

It is imperative to develop MAS-friendly markers that are very tightly linked to the gene of interest (Foolad et al. 2008) in order to carry out high-throughput breeding programmes. As the number of $F_{2}$ lines used was 542 , it is expected that the number of lines showing recombination event would not be high enough in the tomato genome to narrow the genetic and physical interval. Although we have defined the interval to a $900 \mathrm{~kb}$ region, the $900 \mathrm{~kb}$ size of the interval means that there is the possibility of internal recombination events unlinking the marker from the causal locus, which may prove difficult to carry out large-scale breeding programmes. Therefore, using the NGS data from parents and the bulks, we mined the data for single-nucleotide polymorphisms (SNPs) in an attempt to identify the locus with finer resolution. We examined only the $900-\mathrm{kb}$ interval region and a total of 1099 single-nucleotide sites found to be polymorphic (Supplementary Table 1). A heatmap for the polymorphisms was produced (Supplementary Figure 2). We converted three of these single-nucleotide polymorphisms (SNPs) to CAPS markers, 4876, 4942 and $5023 \mathrm{~K}$, and used them to map $\mathrm{Frl}$. They all co-segregate with $\mathrm{Frl}$ (Table 1), confirming the genetic interval and the molecular polymorphism.
Table 1 Segregation of locus among $\mathrm{F}_{2}$ lines that were critical to the mapping of $\mathrm{Frl}$

\begin{tabular}{llllllll}
\hline \multicolumn{2}{l}{ Frl interval on chromosome 9} & & & & \\
\hline F $_{2}$ Lines* & $4206 \mathrm{~K}$ & $4876 \mathrm{~K}$ & $4942 \mathrm{~K}$ & Frl & $5023 \mathrm{~K}$ & $5108 \mathrm{~K}$ & $5190 \mathrm{~K}$ \\
\hline 26 & SS & SS & SS & SS & SS & RS & RS \\
220 & SS & SS & SS & SS & SS & RS & RS \\
247 & RS & RS & RS & R & RS & SS & SS \\
261 & RS & RS & RS & R & RS & RS & RR \\
321 & RS & SS & SS & SS & SS & SS & SS \\
349 & SS & SS & SS & SS & SS & RS & RS \\
377 & RS & SS & SS & SS & SS & SS & SS \\
439 & RS & SS & SS & SS & SS & SS & SS \\
560 & RS & SS & SS & SS & SS & SS & SS \\
518 & SS & SS & SS & SS & SS & RS & RS \\
\hline
\end{tabular}

$* \mathrm{~F}_{2}$ lines were generated from the cross between the resistant and the susceptible cultivars. $S S$ homozygous for susceptible parent allele; $R R$ homozygous for resistant parent allele; $R S$ heterozygous. Important recombinants are given in bold
Fig. 2 Map interval of Frl on tomato chromosome 9. Position of molecular markers used to map the $\mathrm{Frl}$ locus on the reference tomato genome. Numbers below the bar indicate the number of recombinants in 542 $\mathrm{F}_{2}$ individuals

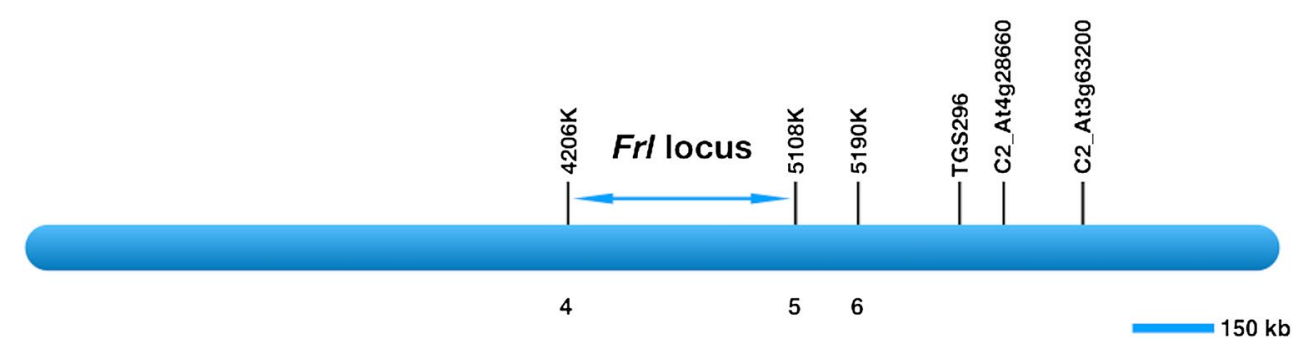




\section{Marker co-segregating with Frl differentiates commercial varieties}

The ultimate aim of any marker development effort is effective use in breeding programmes that include backcrossing, gene pyramiding, eliminating unsuitable lines and accelerated line developments (Collard and Mackill 2008). With this in mind, we have evaluated our newly developed markers against 50 commercial tomato varieties, 24 of which are with known $\mathrm{Frl}$ phenotypes, obtained as seeds. We grew them to seedlings and isolated DNA. We then performed PCR with co-segregating molecular markers. One of our markers was polymorphic with all the $\mathrm{F}_{1} \mathrm{~s}$ and co-segregated with the claimed phenotypes. Representatives of these varieties and their claimed phenotype and the Frl genotype with the marker are given in Table 2. This indicates that the identified polymorphism has been maintained across different varieties during independent breeding programmes and, thus, the marker is tightly associated with FORL resistance and may be close enough to the causal genetic element for resistance to be useful in breeding programmes.

\section{The Frl interval contains defense-related genes}

We attempted to identify gene candidates that could be causally linked to the resistance phenotype, using the annotations of the tomato reference genome (Tomato Genome Consortium 2012). The $F r l$ interval contains a total of 107 predicted genes according to the International Tomato Annotation Group (ITAG) version 3.2 (Supplementary Table 2). Seven of these genes have been annotated as glutathione S-transferase, nine of them as glutathione S-transferase-like

Table 2 Phenotype of commercial tomato varieties and their genotype with the Frl marker developed in this study

\begin{tabular}{lll}
\hline Commercial variety $^{\mathrm{a}}$ & Claimed phenotype $^{\mathrm{b}}$ & $\begin{array}{l}\text { Genotype } \\
\text { with } \text { Frl } \\
\text { marker }\end{array}$ \\
\hline Alberty $\mathrm{F}_{1}$ & Resistant & RS \\
Corvette $\mathrm{F}_{1}$ & Resistant & RS \\
Avalantino $\mathrm{F}_{1}$ & Resistant & $\mathrm{RS}$ \\
Alyanak $\mathrm{F}_{1}$ & Resistant & $\mathrm{RS}$ \\
Akın F1 & Resistant & $\mathrm{RS}$ \\
Çikoköy $\mathrm{F}_{1}$ & Resistant & $\mathrm{RS}$ \\
Seval $\mathrm{F}_{1}$ & Resistant & $\mathrm{RS}$ \\
Vertigo $\mathrm{F}_{1}$ & Susceptible & $\mathrm{SS}$ \\
Moda $\mathrm{F}_{1}$ & Susceptible & $\mathrm{SS}$ \\
Pony Express $\mathrm{F}_{1}$ & Susceptible & $\mathrm{SS}$ \\
\hline
\end{tabular}

${ }^{\text {a }}$ These are selected from 50 readily available varieties on the market

'Phenotype information has been obtained from the companies' websites, which sell these varieties to growers

$R S$ resistant/susceptible heterozygous, $S S$ susceptible homozygous proteins and two of them as putative glutathione S-transferase T2 proteins. Interestingly, these 18 genes (Table 3) are clustered in the centre of the interval as a gene family around the co-segregating marker. In addition to these genes, the interval also contains other defence-related genes including lectin receptor kinase, leucine-rich repeat-containing protein, serine/threonine-protein kinase and kinase family protein (Table 3).

\section{Discussion}

Tomato breeding efforts have been carried out since 1930s and as the technology in molecular biology developed, the use of molecular markers and genetic maps has contributed enormously to the tomato crop improvement (Foolad and Panthee 2012). Using the current NGS technology, we demonstrate evidence that it is possible to generate MASfriendly markers tightly associated with FORL resistance locus ( $F r l)$ in tomato. The $F r l$ locus was originally identified in a mutant of wild-type tomato $S$. peruvianum (also known as Peruvian nightshade). It has been introgressed into the cultivated tomato varieties (Foolad and Panthee 2012)

Table 3 Defence-related genes within $\mathrm{Frl}$ interval

\begin{tabular}{ll}
\hline Gene ID & Putative function \\
\hline Solyc09g011027.1.1 & Pathogenesis-related thaumatin family protein \\
Solyc09g011060.2.1 & Clade IV lectin receptor kinase \\
Solyc09g011070.1.1 & clade XI lectin receptor kinase \\
Solyc09g011235.1.1 & Leucine-rich repeat-containing protein \\
Solyc09g011320.3.1 & Serine/threonine-protein kinase \\
Solyc09g011330.2.1 & Serine/threonine-protein kinase \\
Solyc09g011490.3.1 & Glutathione S-transferase-like protein \\
Solyc09g011500.3.1 & Glutathione S-transferase-like protein \\
Solyc09g011510.2.1 & Glutathione S-transferase-like protein \\
Solyc09g011520.3.1 & Glutathione S-transferase-like protein \\
Solyc09g011530.2.1 & Glutathione S-transferase-like protein \\
Solyc09g011535.1.1 & Glutathione S-transferase-like protein \\
Solyc09g011540.2.1 & Glutathione S-transferase \\
Solyc09g011550.2.1 & Glutathione S-transferase \\
Solyc09g011560.2.1 & Glutathione S-transferase \\
Solyc09g011570.3.1 & Glutathione S-transferase-like protein \\
Solyc09g011580.2.1 & Glutathione S-transferase-like protein \\
Solyc09g011590.3.1 & Glutathione S-transferase-like protein \\
Solyc09g011600.3.1 & Glutathione S-transferase \\
Solyc09g011610.3.1 & Glutathione S-transferase \\
Solyc09g011620.1.1 & Glutathione S-transferase \\
Solyc09g011630.3.1 & putative glutathione S-transferase T2 \\
Solyc09g011640.4.1 & Putative glutathione S-transferase T2 \\
Solyc09g011650.3.1 & Glutathione S-transferase \\
Solyc09g011750.3.1 & Kinase family protein \\
\hline &
\end{tabular}


and has been used in many breeding programmes. Previous mapping exercises have placed $\mathrm{Frl}$ on the chromosome 9 closely linked to the Tm-2 $2^{2}$ gene (Vakalounakis et al. 1997). Several groups have used different approaches to generate PCR-based markers including RAPD (Fazio et al. 1999) and SCAR (Mutlu et al. 2015) markers. However, to our knowledge, the use of these markers in breeding programmes has not been reported. In addition, in our own research selection for $\mathrm{Frl}$ in the tomato breeding proved difficult as these markers were not reliable, most likely due to not being tightly linked to the causal locus. This prompted us to initiate the present investigations, where we generated a mapping population between a resistant and a susceptible parent and used it to identify the tightly associated markers. Bulk segregant analysis (Michelmore et al. 1991) for gene mapping has been used in many different crop species as well as in microbial pathogens. Although the technique was originally used with RAPD markers, since then, it has been widely used in combination with the NGS technology (Devran et al. 2015; Woods-Tör et al. 2018). We utilized this advantage and sequenced the genomic DNA from resistant and susceptible bulks as well as from the parents. We mapped the raw sequences onto the published tomato reference genome and identified SNPs. Subsequently, a few SNPs within $1.2 \mathrm{Mb}$ region of $\mathrm{Frl}$ locus were converted into PCR-based CAPS markers and the $\mathrm{Frl}$ was fine mapped to a $900 \mathrm{~kb}$ interval using $542 \mathrm{~F}_{2}$ lines.

When interval is large, it is recommended that the selection of a trait is carried out with two flanking makers to maximize the probability of success (Collard and Mackill 2008). However, for large-scale breeding programmes, this may not be suitable as it becomes labour intensive. To overcome this and generate co-segregating markers, we searched the entire interval for SNVs and identified 1099 polymorphic sites. Generating PCR-based markers from some of these SNPs enabled us to develop new markers. As the generation of pure lines involves many crossings and selfings, the chances of a recombination event increase with each additional crossing and, thus, tight linkage of marker becomes more important (Yan et al. 2017). Testing our markers on commercial varieties that had been independently developed showed that these new markers also co-segregated with $\mathrm{Frl}$ phenotype, indicating that the marker generated in this study is very tightly linked to $\mathrm{Frl}$ and applicable to multiple varieties.

We showed that $\mathrm{Frl}$ interval contains defence-related genes (Table 3). It is well known that serine/threonineprotein kinase encoding genes such as PTO and PTI are involved in resistance to plant pathogens (Zhou et al. 1995). Similarly, lectin receptor kinase proteins have been reported to be involved in innate immunity in plants (Singh and Zimmerli 2013). Most intriguing was the presence of genes encoding the glutathione S-transferase (GST) family proteins within the interval. GSTs are abundant proteins encoded by a highly divergent ancient gene family and represent a major group of detoxification enzymes (Edwards et al. 2000). It is well known that during fungal infections, oxidative stress is induced and the GSTs contribute to the defence response (Gullner and Komives 2006). There were 18 genes as a cluster encoding them in the vicinity of the co-segregating markers. Interestingly, using a proteomic approach, Mazzeo et al. (2014) have reported the increased level of a GST-like protein, Solyc09g011590 in FORL-resistant tomato plant. It is tempting to speculate that some of these GSTs may be responsible for FORL resistance.

Author contribution statement ZD and MT planned and designed the research. EK and ZD conducted the laboratory work. YH, MT and DJS analyzed the data. MT, YH and DJS interpreted the data and wrote the manuscript.

\section{Compliance with ethical standards}

Conflict of interest The authors declare that there is no conflict of interests.

Funding The research was supported by Multi Tohum A.Ş. (Antalya, Turkey) and M.Y. Genetik Tarım Tek. Lab. Tic.Ltd.Şti. (Antalya, Turkey).

Open Access This article is distributed under the terms of the Creative Commons Attribution 4.0 International License (http://creativeco mmons.org/licenses/by/4.0/), which permits unrestricted use, distribution, and reproduction in any medium, provided you give appropriate credit to the original author(s) and the source, provide a link to the Creative Commons license, and indicate if changes were made.

\section{References}

Baysal Ö, Lai D, Xu HH et al (2013) A proteomic approach provides new insights into the control of soil-borne plant pathogens by Bacillus species. PLoS ONE 8(1):e53182. https://doi.org/10.1371/ journal.pone.0053182

Collard BCY, Mackill DJ (2008) Marker-assisted selection: an approach for precision plant breeding in the twenty-first century. Philos Trans R Soc Lond B Biol Sci 363:557-572. https://doi. org/10.1098/rstb.2007.2170

Devran Z, Kahveci E, Özkaynak E et al (2015) Development of molecular markers tightly linked to Pvr4 gene in pepper using next-generation sequencing. Mol Breed 35:101. https://doi.org/10.1007/ s11032-015-0294-5

Edel-Hermann V, Gautheron N, Steinberg C (2011) Genetic diversity of Fusarium oxysporum and related species pathogenic on tomato in Algeria and other Mediterranean countries. Plant Pathol 61:787-800. https://doi.org/10.1111/j.1365-3059.2011.02551.x

Edwards R, Dixon DP, Walbot V (2000) Plant glutathione S-transferases: enzymes with multiple functions in sickness and in health. Trends Plant Sci 5:193-198. https://doi.org/10.1016/S1360 $-1385(00) 01601-0$

Fazio G, Stevens MR, Scott JW (1999) Identification of RAPD markers linked to Fusarium crown and root rot resistance $(\mathrm{Frl})$ in tomato. Euphytica 105:205-210. https://doi.org/10.1023/A:1003497719 705 
Foolad MR, Panthee DR (2012) Marker-assisted selection in tomato breeding. Crit Rev Plant Sci 31:93-123. https://doi. org/10.1080/07352689.2011.616057

Foolad MR, Merk HL, Ashrafi H (2008) Genetics, genomics and breeding of late blight and early blight resistance in tomato. Crit Rev Plant Sci 27:75-107. https://doi.org/10.1080/07352680802147353

Gullner G, Komives T (2006) Defense reactions of infected plants: roles of glutathione and glutathione S-transferase enzymes. Acta Phytopathologica et Entomologica Hungarica 41:3-10. https:// doi.org/10.1556/APhyt.41.2006.1-2.2

Li H, Durbin R (2009) Fast and accurate short read alignment with Burrows-Wheeler transform. Bioinformatics 25:1754-1760. https ://doi.org/10.1093/bioinformatics/btp324

Li H, Handsaker B, Wysoker A et al (2009) The sequence alignment/ map format and SAMtools. Bioinformatics 25:2078-2079. https ://doi.org/10.1093/bioinformatics/btp352

Lievens B, van Baarlen P, Verreth C et al (2009) Evolutionary relationships between Fusarium oxysporum f. sp. lycopersici and $F$. oxysporum f. sp. radicis-lycopersici isolates inferred from mating type, elongation factor-1alpha and exopolygalacturonase sequences. Mycol Res 113:1181-1191. https://doi.org/10.1016/j. mycres.2009.07.019

Manzo D, Ferriello F, Puopolo G et al (2016) Fusarium oxysporum f.sp. radicis-lycopersici induces distinct transcriptome reprogramming in resistant and susceptible isogenic tomato lines. BMC Plant Biol 16:53. https://doi.org/10.1186/s12870-016-0740-5

Mazzeo MF, Cacace G, Ferriello F et al (2014) Proteomic investigation of response to FORL infection in tomato roots. Plant Physiol Biochem 74:42-49. https://doi.org/10.1016/j.plaphy.2013.10.031

Michelmore RW, Paran I, Kesseli RV (1991) Identification of markers linked to disease-resistance genes by bulked segregant analysis: a rapid method to detect markers in specific genomic regions by using segregating populations. Proc Natl Acad Sci USA 88:98289832. https://doi.org/10.1073/pnas.88.21.9828

Mutlu N, Demirelli A, Ilbi H, Ikten C (2015) Development of co-dominant SCAR markers linked to resistant gene against the Fusarium oxysporum f. sp. radicis-lycopersici. Theor Appl Genet 128:17911798. https://doi.org/10.1007/s00122-015-2547-4

Neff MM, Turk E, Kalishman M (2002) Web-based primer design for single nucleotide polymorphism analysis. Trends Genet 18:613615. https://doi.org/10.1016/S0168-9525(02)02820-2

Robinson JT, Thorvaldsdóttir H, Winckler W et al (2011) Integrative genomics viewer. Nat Biotechnol 29:24-26. https://doi. org/10.1038/nbt. 1754

Saremi H, Saremi H, Okhovvat SM (2008) Major Fusarium diseases on crops and their control management with soil solarisation in northwest Iran. Commun Agric Appl Biol Sci 73:189-199
Singh P, Zimmerli LZ (2013) Lectin receptor kinases in plant innate immunity. Front Plant Sci. https://doi.org/10.3389/ fpls.2013.00124

Szczechura W, Staniaszek M, Habdas H (2013) Fusarium oxysporum f. sp. radicis-lycopersici-the cause of fusarium crown and root rot in tomato cultivation. J Plant Prot Res 53:172-176. https://doi. org/10.2478/jppr-2013-0026

Tomato Genome Consortium (2012) The tomato genome sequence provides insights into fleshy fruit evolution. Nature 485:635-641. https://doi.org/10.1038/nature11119

Truong H, Choi HS, Cho MC, Lee HE (2011) Conversion of the random amplified polymorphic DNA (RAPD) marker UBC\#116 linked to Fusarium crown and root rot resistance gene $(\mathrm{Frl})$ into a co-dominant sequence characterized amplified region (SCAR) marker for marker-assisted selection of tomato. Afr J Biotech 10:11130-11136. https://doi.org/10.5897/AJB11.1520

Vakalounakis DJ, Laterrot H, Moretti A et al (1997) Linkage between Frl (Fusarium oxysporum f.sp. radicis-lycopersici resistance) and Tm-2 (tobacco mosaic virus resistance-2) loci in tomato (Lycopersicon esculentum). Ann Appl Biol 130:319-323. https://doi. org/10.1111/j.1744-7348.1997.tb06835.x

Woods-Tör A, Studholme DJ, Çevik V et al (2018) A suppressor/avirulence gene combination in Hyaloperonospora arabidopsidis determines race specificity in Arabidopsis thaliana. Front Plant Sci 9:1957. https://doi.org/10.3389/fpls.2018.00265

$\mathrm{Xu}$ L, Nonomura T, Suzuki S et al (2006) Symptomatic evidence for differential root invasion by Fusarium crown and root rot pathogens between common tomato Lycopersicon esculentum and its varieties. J Phytopathol 154:577-586. https://doi.org/10.111 1/j.1439-0434.2006.01149.x

Yan G, Liu H, Wang H et al (2017) Accelerated generation of selfed pure line plants for gene identification and crop breeding. Front Plant Sci 8:1786. https://doi.org/10.3389/fpls.2017.01786

Yemataw Z, Muzemil S, Ambachew D et al (2018) Genome sequence data from 17 accessions of Ensete ventricosum, a staple food crop for millions in Ethiopia. Data Brief 18:285-293. https://doi. org/10.1016/j.dib.2018.03.026

Zhao S, Chen X, Deng S et al (2016) The effects of fungicide, soil fumigant, bio-organic fertilizer and their combined application on chrysanthemum Fusarium wilt controlling, soil enzyme activities and microbial properties. Molecules 21:526. https://doi. org/10.3390/molecules21040526

Zhou J, Loh Y-T, Bressan RA, Martin GB (1995) The tomato gene Ptil encodes a serine/threonine kinase that is phosphorylated by Pto and is involved in the hypersensitive response. Cell 83:925-935. https://doi.org/10.1016/0092-8674(95)90208-2 